\title{
Steuern in der Frühen Neuzeit
}

\subsection{Steuern im Heiligen Römischen Reich Deutscher Nation}

\subsubsection{Das Heilige Römische Reich Deutscher Nation}

Seit dem 11. und 12. Jahrhundert hatte sich in Deutschland allmählich eine von der kaiserlichen Zentralgewalt weitgehend unabhängige Landesherrschaft herausgebildet. Diesen Zustand hatte Kaiser Friedrich II. durch die Fürstenprivilegien von 1220 und 1231-1232 bis zu einem gewissen Grad anerkannt. Den Fürsten wurden in der Goldenen Bulle von 1356 ausdrücklich weitere Vorrechte zugesprochen, auch verschiedene Städte nahmen für sich derartige Rechte in Anspruch. Die weltlichen und geistlichen Fürsten beharrten ihrerseits nachdrücklich auf ihrer Selbstständigkeit. Auf diese Weise gelang es den Territorialfürsten seit dem 14. und 15. Jahrhundert, die Landesherrschaft zur Landeshoheit auszubauen und gegen zentralistische Bestrebungen zu verteidigen. Die Folge war, dass sich in Deutschland, im Unterschied zu Frankreich oder England, keine Zentralgewalt etablieren konnte. ${ }^{92}$

Die Versuche, ein System direkter und indirekter Reichssteuern einzuführen, scheiterten an der erreichten Selbstständigkeit und Stärke der Fürsten, der Ritter und der Städte gegenüber der geschwächten Reichsgewalt. Die Reichsstädte und

92 Sahm, Reiner: Zum Teufel mit der Steuer! 5000 Jahre Steuern - ein langer Leidensweg der Menschheit, Wiesbaden 2012, S. 83. 
die Bischofsstädte leisteten dem deutschen Königtum jedoch außerordentliche steuerartige Beihilfen, besonders seit dem 13. Jahrhundert in Form von regelmäBigen „Städtesteuern“.933

Im 15. Jahrhundert kam es zwischen dem Kaiser, den Fürsten und den Städten immer wieder zu Verhandlungen über eine Reform der Reichsverfassung. Dabei spielte neben der Gliederung des Reichsgebietes in überregionale Kreise, einer Reichsgerichtsorganisation, dem Landfrieden und der Aufstellung eines Reichsheeres die Erhebung von Reichssteuern eine besondere Rolle. Die Notwendigkeit zeigte sich vor allem, als es darum ging, für den Kreuzzug gegen die Hussiten und später gegen die Türken - ein schlagkräftiges Reichsheer aufzustellen. Die veränderte militärische Technik - der Einsatz von Schusswaffen - und der größere Bedarf an Fußtruppen machten die Wehrerfassung immer unzureichender und Söldnerheere notwendig. Dazu bedurfte es erheblicher Geldmittel, die in dem Versuch mündeten, die Reichsuntertanen mit einer direkten Reichssteuer - dem „Gemeinen Pfennig" - zu belegen. ${ }^{44}$

Der „Gemeine Pfennig“ war die erste allgemeine Reichssteuer in der deutschen Geschichte und zugleich der Anfang einer Reichsverwaltung in Form einer Steuerbehörde, die im dualistischen Sinn der königlichen Verwaltung gegenübertrat. ${ }^{95}$ Diese Geldsteuer sollte jeden Reichsangehörigen beiderlei Geschlechts, der älter als 15 Jahre war, unmittelbar belasten. Nach der Verordnung des Freiburger Reichsabschieds von 1498 durfte „weder Geistlicher noch Edelmann, Mittelbarer oder Unmittelbarer, Befreiter oder Unbefreiter [...] sich dieser Abgabe entziehen und die Unterthanen konnten mit Gewalt darzu gezwungen werden. ${ }^{\text {“96 }}$ Für die Christen war es eine Synthese aus Kopf-, Vermögen-, Einkommen- und ständischer Personalsteuer, für die Juden eine weitaus höhere Kopfsteuer. ${ }^{97}$

Aufgrund der schwachen Reichsgewalt und der schlecht ausgebauten Reichsverwaltung blieb der "Gemeine Pfennig" ohne nennenswerten Erfolg. Der permanente Widerstand einzelner Reichsstände gegen die Reichssteuern konnte nicht gebrochen werden, sodass der „Gemeine Pfennig“ zwischen 1427 und 1551 wohl elfmal

93 Wagner, Adolph: Finanzwissenschaft 3. Teil, Bd. 1: Steuergeschichte vom Altertum bis zur Gegenwart, Leipzig 1910, überarbeitet und erweitert in Gemeinschaft mit Hermann Deite, ND Glashütten im Taunus 1973, S. 47 ff.

94 Sahm, Reiner: 5000 Jahre Steuern, S. 85.

95 Moraw, Peter: Der „Gemeine Pfennig“. Neue Steuern und die Einheit des Reiches im 15. und 16. Jahrhundert in: Schultz, Uwe (Hrsg.): Mit dem Zehnten fing es an. Eine Kulturgeschichte der Steuer, München 1986, S. 130-142, S. $136 \mathrm{ff}$.

96 Lang, Karl Heinrich von: Historische Entwicklung der Teutschen Steuerverfassungen seit der Karolinger bis auf unsere Zeiten, Berlin/Stettin 1793, ND Aalen 1966, S. 184.

97 Moraw, Peter: Der „Gemeine Pfennig“, S. 137. 
bewilligt und ausgeschrieben, aber nicht ein einziges Mal vollständig erhoben wurde. Er kam zu früh, denn mit diesem einfachen Steuersystem ließen sich die unzähligen politischen, rechtlichen und sozialen Verschiedenheiten, die zu dieser Zeit in Deutschland herrschten, nicht erfassen. Mit dem "Gemeinen Pfennig“ wurde zu tief in die territorialen Belange der Fürsten eingegriffen, die ebenso wie die anderen Stände und die "Steuerbürger" eine solche Steuer als Zumutung empfanden. Es kam deshalb zum Ende dieses Steuersystems mit einem letzten „Pfennig" im Jahre 1551.98

Das Scheitern eines selbstständigen Reichssteuersystems in einer Zeit, in der Territorien in einem größeren Staatskörper nicht mehr ohne Steuern auskamen, erklärt es, dass man notgedrungen zu einem System von Matrikularumlagen im Reich gelangte. Die einzelnen Reichsstände übernahmen dabei bestimmte Summen, die sie auf ihre Untertanen als Beitrag zu den Reichskosten verteilten, und ließen sich dies von ihren Landständen bewilligen. Daraus bildete sich als endgültige Einrichtung das System der sogenannten Römermonate, so genannt nach den nicht mehr stattfindenden Romfahrten. Bei einer drohenden Gefahr oder beispielsweise als Beihilfe zu den Türkenkriegen forderte man die entsprechende Anzahl „Römermonate“ an, die dann nach dem in der Reichsmatrikel festgelegten Schlüssel umgelegt wurden. ${ }^{99}$

Das Steuersystem des Reiches ist über die Stufe der außerordentlichen Steuern nicht hinausgekommen. Die Steuerdiskussionen in der ersten Hälfte des 16. Jahrhunderts drehten sich nicht um die Frage der prinzipiellen Berechtigung von Steuern, sondern ausschließlich um den Gemeinen Pfennig und um die Matrikel; es ging im Wesentlichen um Fragen der Höhe und der Verwendung der Steuern, worin man einen Schritt in Richtung Steuerstaat sehen kann. ${ }^{100}$ Die Reichsstände entschieden als Verband auf den Reichstagen autonom über die Bewilligung, Durchführung und Verwendung der Gelder, dem Reich wurde keine Steuerhoheit zugestanden. ${ }^{101}$

98 Wagner, Adolph: Finanzwissenschaft 3. Teil, S. 53; Moraw, Peter: Der „Gemeine Pfennig“, S. 136-141; Schomburg, Walter: Lexikon der deutschen Steuer und Zollgeschichte. Abgaben, Dienste, Gebühren, Steuern und Zölle von den Anfängen bis 1806, München 1992, S. 120 f.; Sahm, Reiner: 5000 Jahre Steuern, S. 85.

99 Wagner, Adolph: Finanzwissenschaft 3. Teil, S. 55 f.

100 Schmid, Peter: Reichssteuern, Reichsfinanzen und Reichsgewalt in der ersten Hälfte des 16. Jahrhunderts, in: Angermeier, Heinz/Seybold, Reinhard (Hrsg.): Säkulare Aspekte der Reformationszeit, München/Wien 1983, S. 153-198, S. 196.

101 Ebd., S. 197. 


\subsubsection{Die deutschen Territorien und Einzelstaaten}

In den geistlichen und weltlichen Territorien und Einzelstaaten lag bei der allgemein vorherrschenden Naturalwirtschaft der Schwerpunkt der Leistungen jahrhundertelang in persönlichen Dienstpflichten und Naturallieferungen. Daneben wurde seit dem 12. Jahrhundert in allen deutschen Territorien die sogenannte Bede, die ursprünglich den Vögten, Grafen und Fürsten nur unregelmäßig und mehr oder weniger freiwillig geleistet wurde, als regelmäßig anfallende direkte Steuer erhoben. ${ }^{102}$ Nach Fälligkeitsterminen wurde die „ordentliche Bede“ ein- bis dreimal jährlich erhoben und lastete auf dem Grundbesitz der einzelnen Bürger und Bauern. ${ }^{103}$

Überwiegend wurde die Bede dann gefordert, wenn es sich um eine Beihilfe zur Kostendeckung von solchen „öffentlichen“ Leistungen handelte, welche die Herrschenden von Amts wegen auszuführen hatten; dazu zählte vor allem der Heerdienst. Da dieser Heerdienst von dem Landesherrn aus den gewöhnlichen domanialen und anderen Einkünften zu leisten war, bestand die Bede aus einer nur auf „Bitte“ zu leistenden, im Laufe der Zeit aber eingeforderten ordentlichen Steuer. ${ }^{104}$

\subsubsection{Die deutschen Städte}

Der staatspolitische Werdegang in Deutschland brachte es mit sich, dass die schwach ausgebildete Zentralgewalt sich nicht des Steuerwesens bemächtigen konnte, sondern zulassen musste, dass es eine nahezu ausschließliche Angelegenheit der Stände, insbesondere der Territorialherrscher und der städtischen Obrigkeiten, wurde. ${ }^{105}$

In den städtischen Kommunen, in denen sich ein eigenes Stadtrecht, eine selbstständige Verwaltung sowie eine Wehr- und Finanzhoheit herausgebildet hatten, wurden die Bürger auf vielfache Weise zur Finanzierung der kommunalen Aufgaben herangezogen. Da sind an erster Stelle die Bedürfnisse der Bürger nach Schutz vor Angriffen von außen zu nennen, die eine Umfriedung des Stadtgebietes durch Befestigungsanlagen sowie die Bereitstellung der entsprechenden Bewaffnung erforderten. Des Weiteren gehörte zur Stadt ein repräsentatives Verwaltungszentrum. Sodann musste eine Infrastruktur - Straßen, Wege, Brücken - angelegt werden, es waren die Wasserver- und -entsorgung zu sichern, Markteinrichtungen zu schaffen usw. Hinzu kamen der Bau von Schulen und die Einrichtungen für das Gesund-

102 Wagner, Adolph: Finanzwissenschaft 3. Teil, S. 67.

103 Schomburg, Walter: Lexikon, S. 36.

104 Wagner, Adolph: Finanzwissenschaft 3. Teil, S. 76.

105 Sahm, Reiner: 5000 Jahre Steuern, S. 87. 
heitswesen und für die Armenpflege. Schließlich galt es, dafür Sorge zu tragen, dass die Einwohnerschaft ihren religiösen Bedürfnissen nachgehen konnte. Die Städte hatten auch Wirtschaftseinrichtungen - Teiche, Vorwerke, Mühlen, Schlachthöfe, Badestuben, Gasthäuser, Brauereien - vorzuhalten, die sie in der Regel an Bürger verpachteten. Verschiedene Städte hatten, um die Nahrungsmittelversorgung der Bürger abzusichern, ganze Dörfer im Umland erworben. ${ }^{106}$

Darüber hinaus wurden von den Städten finanzielle Beiträge gefordert, die nicht in unmittelbarem Zusammenhang mit innerstädtischen Angelegenheiten standen. Dazu gehörten die „Städtesteuern“, die die Reichs- und die Bischofsstädte an die Zentralgewalt zu entrichten hatten. Ebenso hatten die Freien Reichsstädte, die seit 1489 auf den Reichstagen eine geschlossene Kurie bildeten, sich an den Matrikularumlagen zu beteiligen wie die Reichsstädte, die auf dem Krongut errichtet worden waren. Die landesherrlichen Städte hatten den finanziellen Forderungen der Territorialherren zu entsprechen. ${ }^{107}$

Um sowohl die innerstädtischen als auch die von außen an die Kommunen herangetragenen Aufgaben finanzieren zu können, waren die Stadträte genötigt, sowohl von den Bürgern finanzielle Beiträge einzuziehen als auch Erträge städtischer Wirtschaftseinrichtungen einzusetzen. Diese neuen öffentlich-rechtlichen Verpflichtungen hatten zum ersten Mal in Deutschland die Natur wirklicher Steuern. Gierke ${ }^{108}$ führt mit zahlreichen Beispielen den Nachweis, warum in den Städten zuerst von eigentlichen „Steuern“ und von der Steuerpflicht des Bürgers gesprochen werden kann.

Die städtischen Steuern waren die ersten Beiträge, welche ein Gemeinwesen allein um des öffentlichen Wohls willen von seinen Gliedern als solchen forderte. Einziger und zureichender Grund für die Erhebung einer städtischen Steuer war das städtische Bedürfniß [...], und verpflichtet zur Tragung der Steuer waren alle Bürger und nur die Bürger allein um deshalb, weil sie Bürger waren. [...] An sich war daher auch die Steuerpflicht jedes vollberechtigten Bürgers gleich. Aber diese Pflicht bestand darin, nach Verhältniß und Vermögen beizutragen. ${ }^{109}$

Die Stadt trat den Einzelnen als höhere Person des öffentlichen Rechts gegenüber, sie war die Quelle der amtlichen Machtbefugnisse. So entwickelte sich die Stadt zu

106 Sahm, Reiner: 5000 Jahre Steuern, S. $87 \mathrm{f}$.

107 Ebd., S.88.

108 Gierke, Otto: Das deutsche Genossenschaftsrecht, Bd. 2: Geschichte des deutschen Körperschaftsbegriffs, Berlin 1873, S. $698 \mathrm{ff}$.

109 Ebd., S. 698. 
einer eigenen selbständigen einheitlichen Finanzhoheit, die anerkannte Vorhaben zu erfüllen hatte und dies mit Hilfe von Einnahmen aus Steuern auch tat. ${ }^{110}$

Die indirekten Steuern spielten zunächst eine maßgebliche Rolle. Sie wurden auf Waren erhoben, die von der Bürgerschaft unbedingt nachgefragt wurden, also Verzehr- und Genussmittel. Diese Steuern wurden als Ungeld, Zise oder Akzise bezeichnet und sind auch als solche anerkannt und empfunden worden.

Neben diesen indirekten Verbrauch- und Verkehrsteuern gingen die Stadträte dazu über, bei den Wohlhabenderen auch das Vermögen und das Einkommen zu besteuern, wobei die wirtschaftliche Leistungsfähigkeit der Bürger berücksichtigt wurde. Um diese festzustellen, vertraute man auf die Steuerehrlichkeit der Bürger, die eine eidliche Selbsteinschätzung vor Steuerkommissionen - daher „Eidsteuer“ - abgeben mussten. ${ }^{111}$

Neben diesen allgemeineren direkten Steuern finden sich in den Städten Realsteuern, besonders Grund- und Gebäudesteuern und teilweise auch Gewerbesteuern. ${ }^{112}$

\subsection{Die ungleichmäßige Verteilung der Steuerlasten}

\subsubsection{Die Bauern im Kampf um Gerechtigkeit}

In Deutschland begannen bäuerliche Unruhen verstärkt Ende des 15. Jahrhunderts und erreichten in den Zwanzigerjahren des 16. Jahrhunderts ihren Höhepunkt. ${ }^{113}$ Wesentliche Ursachen der Unruhen waren einerseits die aus den Kreisen der Theologie in immer weitere Kreise vordringenden Erörterungen über das Naturrecht, das auf der Überzeugung beruht, dass ein Minimum von Rechten den Menschen von Gott aus zu allen Zeiten und unter allen Verhältnissen zustehe. Der Glaube an eine unerschütterliche, unanfechtbare „göttliche Gerechtigkeit“ stand dabei im Gegensatz zu bestehender Leibeigenschaft. ${ }^{114}$

Andererseits empfanden die Bauern die undurchschaubaren feudalrechtlichen Abgaben als zu hoch und ungerecht. ${ }^{115}$ In den Beschwerden und Forderungen der

110 Ebd., S.744f.

111 Wagner, Adolph: Finanzwissenschaft 3. Teil, S. 60-63.

112 Ebd., S.64.

113 Sahm, Reiner: 5000 Jahre Steuern, S. 99.

114 Waas, Adolf: Der Bauernkrieg. Die Bauern im Kampf um Gerechtigkeit 1300 bis 1525, München o. J, S. 57 f. und S. 93.

115 Blickle, Peter: „Der Stürn halb ist unser Beger, uns die zu ringren“, S. 143. 
Untertanen wurde die Summe der Abgaben und Belastungen als schiere Tyrannei dargestellt. In der Tat ist das erste Jahrhundert der Neuzeit von einem bislang ungewöhnlichen Anwachsen der Steuern gekennzeichnet. Die traditionelle Belastung der zahlenmäßig am stärksten und am härtesten betroffenen Landbevölkerung erhöhte sich um ein Vielfaches. Dazu kamen Preiserhöhungen durch indirekte Steuern auf Wein, Bier, Fleisch und Mehl. ${ }^{116}$

Weiteres Leid kam durch die Kriegssteuern als Ersatz für nicht geleistete Heerfahrtspflicht, die von den Obrigkeiten auf die Untertanen umgelegt wurden. Die Erfindung und die Perfektionierung der Feuerwaffen hatten einen tiefen Wandel im Heerwesen verursacht. An die Stelle der alten Vasallenheere traten Söldnerheere, die zu höheren Kriegskosten und damit zu einem dauerhaften Anstieg der steuerlichen Belastungen führten. ${ }^{117}$

In fast allen deutschen Aufstandsgebieten fanden bis April 1525 die zwölf Artikel der oberschwäbischen Bauern Verbreitung ${ }^{118}$ und wurden zu einer Art Bekennerschrift der ganzen Bauernbewegung. In den Artikeln 2, 7, 8 und 11 wird auf die untragbaren Belastungen eingegangen. In Artikel 2 bekennen die Bauern sich zur Zahlung des großen Zehnten - der auf Getreide und Großvieh zu entrichten war -, hingegen lehnten sie den kleinen Zehnten - der auf Früchte und Kleinvieh zu leisten war - ab. In Artikel 7 und 9 wird gefordert, dass der Herr den Bauern keine weiteren Lasten auferlegen soll, insbesondere sollen die untragbaren Pachtzinsen überprüft werden. In Artikel 11 wird gefordert, den „Todfall“ abzuschaffen. ${ }^{119}$

Mit der Opposition der Aufständischen gegen die Abhängigkeiten und die $\mathrm{zu}$ leistenden Steuern und Abgaben wurde das gesamte Bündel der feudalen Belastungen infrage gestellt - und damit das bestehende System als solches. Der Bauernaufstand war zweifellos die größte Massenerhebung in Europa vor der Französischen Revolution und auch der Aufstand mit der weitreichendsten Pers-

116 Sahm, Reiner: 5000 Jahre Steuern, S. 105.

117 Karl Heinrich Lang hat sich 1793 mit der historischen Entwicklung der „Teutschen Steuerverfassungen seit der Karolinger bis auf unsere Zeiten“ beschäftigt. In seiner „Musterrolle der bis zu Anfang des 16ten Jahrhunderts vorkommenden Auflagen und andern Unterthanschuldigkeiten“ führt er Abgaben und Steuern auf, deren Zahl sich auf fast 750 beläuft. Nach seiner Erkenntnis ist „,jede Veränderung in dem System der Auflagen oder dem Steuerwesen [...] in einer vorausgegangenen Veränderung des Kriegswesens ganz sicher zu suchen. "Lang, Karl Heinrich von: Historische Entwicklung, S. 4; Sahm, Reiner: 5000 Jahre Steuern, S. 105.

118 Jonscher, Reinhard: Der Bauernkrieg in Thüringen, Mühlhausen 2003, S. $102 \mathrm{f}$.

119 Waas, Adolf: Der Bauernkrieg, S.95. Bei dem „Todfall“ handelte es sich um das Heimfallrecht, das die Abhängigkeit der Bauern von den Grundherren und dessen grundherrlichem Erbrecht aus früheren Zeiten dokumentieren sollte. 
pektive. ${ }^{120}$ Die Aufständischen wollten eine neue gesellschaftliche und politische Ordnung. Sie wollten nicht nur die Steuerfreiheit der „Herrenstände“ aufheben, sondern wollten auch, dass diese ihre privilegierte politische, wirtschaftliche und soziale Stellung aufgaben. ${ }^{121}$

\subsubsection{Die ungleichmäßige Lastenverteilung}

Im Heiligen Römischen Reich Deutscher Nation war ein zentraler Streitpunkt der „Gemeine Pfennig“. Einerseits entzündete sich die Kritik an der Heranziehung auch der Geistlichkeit und der Fürsten, andererseits an der überproportionalen steuerlichen Belastung vor allem der Kaufleute. ${ }^{122}$ Immer wenn im 16. Jahrhundert dem Kaiser eine Reichssteuer bewilligt wurde, entstand dabei die Frage, ob diese mithilfe des "Gemeinen Pfennigs“ oder mit dem „Römermonat“ zu erheben wäre. Der Kaiser selbst und „die ihm anhängende demokratische Parthie“ stimmten meistens für die Erhebung des „Gemeinen Pfennigs“, der Adel arbeitete aber jederzeit dagegen, da ihm an der Umsetzung der „Römermonate“ gelegen war. Der wesentliche Unterschied beider Steuern ist folgender: Zu dem „Gemeinen Pfennig“"

musste jeder Fürst von seinem Vermögen, so gut wie der Bauer, der Edelmann, der Beamte, der Erzbischof, der Domherr, der Pfarrer, der reiche Kapitalist, wenn er auch kein Haus und Acker hatte, ein seinem Vermögen oder seinem Einkommen angemeßenes Prozent beisteuern, und die Last der Steuermasse fiel mehr auf den reichern, als den ärmern Theil. ${ }^{123}$

Bei dem „Römermonat" hingegen zahlten die Stände, der Adel, die Geistlichen und Beamten nichts, die gesamte Last blieb allein bei den Bürgern und Bauern. „Ja die Vertheilung der Römermonate unter die Stände selbst war immer höchst ungleich und beschwerend und daher eine unversiegliche Quelle von Moderationsgesuchen. ${ }^{\text {"124 }}$

Die Diskussion um den „Gemeinen Pfennig“ erreichte einen entscheidenden Punkt auf dem Reichstag von Speyer im Jahre 1544, als sich der Kurfürsten- und Fürstenrat in dieser Frage spalteten. Letztendlich haben sich die Gegner des „Gemeinen Pfennigs" durchgesetzt, jedoch unter Festschreibung der grundsätzlichen Steuerpflicht aller Reichsuntertanen in der Bestimmung des „Reichsabschieds“

120 Blickle, Peter: „Der Stürn halb ist unser Beger, uns die zu ringren“, S. 145.

121 Ebd., S. $144 \mathrm{f}$.

122 Schmid, Peter: Reichssteuern, Reichsfinanzen und Reichsgewalt, S. $171 \mathrm{f}$.

123 Lang, Karl Heinrich von: Historische Entwicklung, S. 190.

124 Ebd., S. 190. 
von 1548. Mit der gleichzeitig getroffenen Entscheidung für die Reichsmatrikel verlagerte sich, soweit es um den nicht reichsunmittelbaren Adel und Klerus ging, die Diskussion auf die Territorialebene. ${ }^{125}$

Steuern sollten nur eine vorrübergehende Erscheinung bleiben und nur ein letztes Mittel zur Finanzierung des Herrschers darstellen, denn Steuern haftete das Merkmal der politischen Abhängigkeit und der Unfreiheit an. ${ }^{126}$ „Sparsamkeit ist die beste Steuer" - dieses Sprichwort war das charakteristische Steuerideal, das von vielen Autoren aufgegriffen wurde, um auch auf die zunehmend durch Steuern finanzierten Kosten der Hofhaltung hinzuweisen. ${ }^{127}$

In der Literatur des 16. Jahrhunderts werden neue Landessteuern nur dann als gerecht angesehen, wenn diese mit Zustimmung der Untertanen bzw. Stände erhoben werden. Nur dann, wenn ein legitimer Zweck für die Auferlegung neuer Steuern gegeben war - worüber Beratungen mit den Ständen zu pflegen waren -, war eine Steuerforderung des Landesherrn grundsätzlich berechtigt. ${ }^{128}$ Dies gilt nach überwiegender Ansicht dann nicht, wenn Steuern „in höchster lands not“ erhoben werden müssen; in diesem Fall müssen die Untertanen ihre Zustimmung geben. ${ }^{129}$

Der erste große Untertanenprozess, der die literarische Diskussion über Territorialsteuern nachhaltig beeinflusst hat und in der Literatur nachweisbar ist, ist ein sich über mehr als hundert Jahre hinziehender Rechtsstreit der „vier großen Dörfer“ Hatten, Rittershofen, Ober- und Niederbetschdorf im Hattgau gegen den Grafen Hanau-Lichtenberg vor dem Reichskammergericht. ${ }^{130}$ Die Anfänge des Falles reichten bis in den Bauernkrieg zurück. Bei der Auseinandersetzung ging es u. a. um den zu leistenden Huldigungseid, um die Waldnutzung und immer wieder auch um Fragen der Besteuerung. ${ }^{131}$

125 Schwennicke, Andreas: „Ohne Steuer kein Staat“, S. $91 \mathrm{f}$.

126 Schmid, Peter: Reichssteuern, Reichsfinanzen und Reichsgewalt, S. 161.

127 Ebd., S. 41.

128 Wachenhausen, Manfred: Staatsausgabe und Öffentliches Interesse in den Steuerrechtfertigungslehren des naturrechtlichen Rationalismus. Ein dogmengeschichtlicher Beitrag zur Rationalität des Modernen Staates in seinem Verhältnis zum Individuum (Schriften zur Verfassungsgeschichte 16), Berlin 1972, S. $68 \mathrm{ff}$.

129 Schwennicke, Andreas: „Ohne Steuer kein Staat“, S. 65 ff.

130 Bierbrauer, Peter u.a. (Saarbrücker Arbeitsgruppe): Huldigungseid und Herrschaftsstruktur im Hattgau (Elsaß), in: Jahrbuch für westdeutsche Landesgeschichte 6 (1980), S. 117-155, S. 117 f.

131 Ebd., S. 145, Anm. 116. 


\subsubsection{Die Steuerfreiheit der Geistlichkeit}

Im Laufe des 15. Jahrhunderts hatte in den deutschsprachigen Kernlanden des Heiligen Römischen Reiches Deutscher Nation die Unzufriedenheit über die außerordentlich hohen finanziellen Ansprüche der römisch-katholischen Kirche und über die Art und Weise, wie der Klerus mit den erhobenen Finanzmitteln verfuhr, stetig zugenommen. Die römisch-katholische Kirche hatte über die Jahrhunderte ein vielgestaltiges Abgabensystem geschaffen, durch das sich der Klerus einen wesentlichen Anteil an den wirtschaftlichen Ergebnissen, die von den Bauern, den Handwerkern, den Kaufleuten erzielt wurden, aneignen konnte. ${ }^{132}$

Dieses vielschichtige Abgabensystem ermöglichte es der römisch-katholischen Kirche, sowohl ihre religiöse Funktion wahrzunehmen und die dafür erforderlichen materiellen und personellen Bedingungen zu sichern, als auch in einem zunehmenden Maße weltliche Politik zu betreiben. Entsprechend wurde in erster Linie dafür gesorgt, dass ein wachsender Teil der finanziellen Erhebungen direkt der Kammer des Papstes zufiel. ${ }^{133}$

Auch aus dem reformatorischen Umfeld wurde die Steuerfreiheit der Geistlichkeit wie des Adels heftig angegriffen. ${ }^{134}$ Sie widersprach dem Prinzip der Allgemeinheit, weil die Herrenstände Geistlichkeit und Adel von ihr befreit waren, und sie widersprach dem Gleichmäßigkeitsprinzip, weil sie die Bauern neben den Naturalund Geldabgaben an ihre Grundherren über das herkömmliche Maß zusätzlich belastete. ${ }^{135}$ Andererseits oblag dem Klerus z. B. die Fürsorge für Kranke und Arme und die Organisation des Unterrichts- und Bildungswesens. ${ }^{136}$

Eine besondere Belastung für die christliche Bevölkerung war der Zehnt, der vor allem von der Bauernschaft erhoben wurde. Von dem Zehnt sollte jeweils ein Viertel dem Bischof, dem Klerus, den Armen und dem Kirchenbau zukommen. Bäuerliche Wirtschaften, die zu einer kirchlichen Einrichtung gehörten, hatten

132 Sahm, Reiner: 5000 Jahre Steuern, S. 109.

133 Ebd., S. 109.

134 „Dann diser Text trifft furderlicher dann yrgent ein anderer den Adel / und die geystligkeit / Dann wann es geldt und zinße antrifft / so stossen sie die Köpfe zesammen / ziehen sich aus der schlingen / und erdencken also vil list wider das Euangelion / und alle Christenliche brüderschafft / das sie one entgeldt mit geniessen / Ja den besten genieß haben / und alle burde auf die burger pawen und gemeynen man treyben“; Linck, Wentzeslaus, Altenburg o. J. (1516), zitiert in: Schwennicke, Andreas: „Ohne Steuer kein Staat", S. 89.

135 Blickle, Peter: „Der Stürn halb ist unser Beger, uns die zu ringren“, S. 146.

136 Vgl. Reif, Walter: Abweichungen vom Grundsatz der Allgemeinheit der Besteuerung, Nürnberg 1953, S. 47 f.; Davidsohn, Lars: Verfassungsrechtliche Würdigung, S. 12. 
neben dem Zehnten die Grundrente zu entrichten. Der Klerus in den Ländern war dazu berechtigt, verschiedene Gebühren und Abgaben zu erheben. ${ }^{137}$

Die Steuerimmunität des Klerus und der Kirche wurde nicht durch das Gewohnheitsrecht, sondern durch einen Konzilsbeschluss geschaffen. Die Kirche nahm erstmalig auf dem dritten Laterankonzil (1179) grundsätzlich zur Frage der Besteuerung ihrer Güter und Personen durch die weltliche Gewalt Stellung. Nach einer Erneuerung der kirchlichen Steuerimmunitätsgesetzgebung auf dem vierten Laterankonzil (1215) wurde die Bulle "Clericis laicos"von Papst Bonifaz VIII. am 25. Februar 1296 erlassen, nach der eine Auferlegung von weltlichen Steuern auf kirchliches Gut ohne Zustimmung des Papstes verboten wurde. Die Bulle nennt der Reihe nach den Kaiser, Könige, Fürsten, Herzöge, Grafen, Barone, lokale Gewalthaber, Hauptleute, Beamte und die Vorsteher von Städten, denen die Auferlegung solcher Steuern untersagt wurde. Ein Verstoß gegen dieses Verbot wurde mit der Exkommunikation in ihrer schärfsten Form bedroht. ${ }^{138}$

Wegen der Bulle musste Bonifaz VIII. mit Frankreich und England in einen für die Könige siegreichen Kampf eintreten, bei dem es sich im Grunde um die Frage gehandelt hat, ob „bloss der Papst das Recht der geistlichen Besteuerung habe, oder ob dieses Recht auch dem Landsherren zustehe. ${ }^{\text {"139 }}$ Als das Papsttum im Jahr 1305 zum Spielball französischer Machtinteressen wurde, büßte es seine Autorität als überparteiliche Macht ein; die kirchliche Immunität hatte ihren stärksten Schützer verloren. Sie konnte nicht einmal durch Spezialprivilegien gerettet werden. ${ }^{140}$ In Deutschland wurde das Reichsrecht immer mehr durch das lokale Recht abgelöst, was zur Folge hatte, dass Kirchen, Klöster und Kleriker sich mehr oder weniger „an den weltlichen Arm“ anlehnen mussten. Wo die Einsicht in das Bedürfnis der Zeit vorhanden war, kam es zu einer „Angleichung des kirchlichen Elementes an den weltlichen Staatsorganismus. ${ }^{\text {"1411 }}$

In der politischen Praxis und in der juristischen Literatur blieb die Steuerfreiheit von Kirchengut und Klerikern umstritten. Auch in der Rechtsprechung wurde die Steuerfreiheit der Kirche nur einschränkend interpretiert. Es gelang jedoch

137 Sahm, Reiner: 5000 Jahre Steuern, S. 110.

138 Mack, Eugen: Die kirchliche Steuerfreiheit in Deutschland seit der Dekretalenfestsetzung (Kirchliche Abhandlungen 88), Stuttgart 1916, ND Amsterdam 1965, S. 17 ff.

139 Gottlob, Adolf: Die päpstlichen Kreuzzugs-Steuern des 13. Jahrhunderts. Ihre rechtliche Grundlage, politische Geschichte und technische Verwaltung, Heiligenstadt 1892, S. 134.

140 Mack, Eugen: Die kirchliche Steuerfreiheit in Deutschland, S. 245.

141 Ebd., S. 246. 
den Herrschern in Bayern, Österreich, Sachsen und Brandenburg bis zum Ende des 15 . Jahrhunderts, die Kirche an den außerordentlichen Lasten zu beteiligen. ${ }^{142}$

\subsubsection{Die „Steuerpflicht" des Adels}

Schon im 15. Jahrhundert war die Frage, ob überhaupt eine Steuerpflicht des Adels begründet werden kann, Gegenstand heftiger politischer Auseinandersetzungen. Im Hinblick auf die Reichssteuern stellte sich wiederholt die Frage, wie der Adel beteiligt werden sollte. Die Hussitenkriegssteuer von 1427 und die Türkenkriegssteuer von 1471/74 sahen eine Beisteuer der Grafen, Herren und niederen Adligen durch eine ständische Personalsteuer vor. ${ }^{143}$ Andere Reichssteuerprojekte lehnte der Adel ab, da er sich zu unentgoltenen Dienstleistungen im Bereich der Verteidigung verpflichtet fühlte. ${ }^{144}$ Dies änderte sich im 16. Jahrhundert; nach der Ordnung des „Gemeinen Pfennigs" von 1542/44 wurden alle weltlichen und geistlichen Reichsstände und deren Untertanen mit einer Vermögensteuer belastet. ${ }^{145}$

Die Heranziehung des Adels zu den territorialen Steuern war seit Mitte des 16. Jahrhunderts streitig. In der deutschen Literatur wurde einerseits betont, dass der Adel mit seinen Lehen nicht durch Ritterdienste und Steuern doppelt belastet werden dürfe, „dieweil sie dieselbigen mit ihrem leibe verdienen und Ritterdienste davon thun müssen dürffen sie keine stewer am gelde geben. ${ }^{\text {"146 }}$ Andererseits wurde die Meinung vertreten, dass der Adel die an sich gebrachten steuerbaren Bauerngüter weiterhin versteuern müsse und auch steuerpflichtig sei, sofern er städtische Güter besitzt oder Handel treibt. ${ }^{147}$ Eine generelle Steuerfreiheit des Adels - so führt Pistoris aus - sei nirgendwo im Recht bestimmt. Grund für die Steuerbefreiung seien allein die Ritterdienste und nicht die Person oder der Adel an sich. ${ }^{148}$ Sofern Adelige in einer Stadt wohnten, wird die Verbindung von Bürgerrecht und Steuerpflicht dafür

142 Ebd., S. 57 ff. (Bayern), S. 79 ff. (Österreich), S. 107 ff. (Sachsen) und S. 147 ff. (Brandenburg).

143 Isenmann, Eberhard: Reichsfinanzen und Reichssteuern im 15. Jahrhundert, in: Zeitschrift für historische Forschung 7 (1980), S. 1-75 und S. 129-218, S. 158 und S. $163 \mathrm{ff}$.

144 Ebd., S. 186.

145 Ebd., S. 197.

146 Pistoris, Modestinus/Pistoris, Simon/Fachsius, Ludovicus: Consilia sive responsa Trium Saxoniae Iureconsultorum celeberrimorum. Pistoris, Jacob (Hrsg.), Bd. 2, Leipzig 1599, cons. IX, n. 46, S.75; zitiert nach: Schwennicke, Andreas: „Ohne Steuer kein Staat“, S. 98 .

147 Schwennicke, Andreas: „Ohne Steuer kein Staat“, S. $178 \mathrm{f}$.

148 Ebd., S.98f. 
ausschlaggebend gewesen sein, dass man es als gerecht ansah, dass in diesem Fall auch der Adel einen Teil der städtischen Lasten mitzutragen hatte. ${ }^{149}$

Insgesamt kann festgestellt werden, dass die Reichweite der Steuerpflicht und der Rechtsgrund einer Steuerfreiheit des Adels im 16. Jahrhundert weitgehend umstritten und unklar blieben. ${ }^{150}$

\subsection{Zusammenfassung und Fazit}

\section{Zum Grundsatz der Allgemeinheit der Besteuerung}

Der „Gemeine Pfennig“ war die erste allgemeine Steuer in der deutschen Geschichte und sollte als Geldsteuer jeden Reichsangehörigen, der älter als 15 Jahre war, unmittelbar belasten. Damit war in der Frühen Neuzeit - abgesehen von der Belastung der Juden - das Prinzip der Allgemeinheit der Besteuerung grundsätzlich erfüllt, doch blieb der „Gemeine Pfennig“ aufgrund des permanenten Widerstands einzelner Reichsstände gegen diese Reichssteuer ohne nennenswerten Erfolg.

In den deutschen Territorien und Einzelstaaten wurde die "ordentliche Bede“ mehr oder weniger freiwillig geleistet, im Lauf der Zeit wurde diese jedoch als ordentliche Steuer eingefordert und lastete auf dem Grundbesitz der einzelnen Bürger und Bauern.

Die städtischen Steuern waren die ersten Beiträge, die von allen Bürgern allein deshalb, weil sie Bürger waren - zur Erfüllung des öffentlichen Wohls gefordert wurden. Durch die Steuerpflicht aller Bürger - dazu zählten auch die Adeligen - entwickelte sich die Stadt zu einer Finanzhoheit, die die anerkannten Gemeinzwecke erfüllte.

In der politischen Praxis und in der Literatur wurde die Steuerfreiheit von Kirchengut, Klerikern und des Adels heftig angegriffen. Die Steuerimmunität des Klerus und der Kirche wurde durch Konzilsbeschluss geschaffen, und ein Verstoß gegen dieses Verbot wurde mit der Exkommunikation in ihrer schärfsten Form bedroht. Auch in der Rechtsprechung wurde die Steuerfreiheit nur einschränkend interpretiert. Eine generelle Steuerfreiheit des Adels war nirgends im Recht bestimmt. Grund für die reale Steuerbefreiung waren allein die Ritterdienste und nicht der Adel oder die Person an sich.

149 Erler, Adalbert: Bürgerrecht und Steuerpflicht im mittelalterlichen Städtewesen mit besonderer Untersuchung des Steuereides (1939), 2. Aufl., Frankfurt am Main 1963, S. $18 \mathrm{ff}$.

150 Ebd., S. 100. 


\section{Zum Grundsatz der Gleichmäßigkeit der Besteuerung}

Zentraler Streitpunkt im Reich war die Frage, ob mit der Bewilligung einer Reichssteuer durch den Kaiser, diese mithilfe des "Gemeinen Pfennigs" oder mit dem „Römermonat“ zu erheben wäre. Hintergrund dieser Auseinandersetzung war, dass beim "Gemeinen Pfennig“ die Last der Steuermasse auf den reicheren Teil fiel, hingegen bezahlten bei dem „Römermonat" die Stände, der Adel, die Geistlichen und Beamten, nichts. Aufgrund der Machtverhältnisse haben sich die Gegner des „Gemeinen Pfennigs" durchgesetzt, sodass die gesamte Last bei den Bürgern und Bauern blieb. Darüber hinaus widersprach der Kirchenzehnt dem GleichmäBigkeitsprinzip, da dieser die Bauern neben Natural- und Geldabgaben an ihre Grundherren zusätzlich belastete.

\section{Zum Prinzip der Besteuerung nach der Leistungsfähigkeit}

In den Städten war grundsätzlich die Steuerpflicht für jeden Bürger gleich, aber diese Pflicht bestand darin, nach Verhältnis und Vermögen beizutragen. Damit wurde bereits in der Frühen Neuzeit die Leistungsfähigkeit der Bürger zum Maßstab der steuerlichen Belastung. Zu dieser Zeit vertraute man auf die Steuerehrlichkeit der Bürger, die eine eidliche Selbsteinschätzung vor Steuerkommissionen abgeben mussten.

Dieses Buch wird unter der Creative Commons Namensnennung 4.0 International Lizenz (http://creativecommons.org/licenses/by/4.0/deed.de) veröffentlicht, welche die Nutzung, Vervielfältigung, Bearbeitung, Verbreitung und Wiedergabe in jeglichem Medium und Format erlaubt, sofern Sie den/die ursprünglichen Autor(en) und die Quelle ordnungsgemäß nennen, einen Link zur Creative Commons Lizenz beifügen und angeben, ob Änderungen vorgenommen wurden.

Die in diesem Buch enthaltenen Bilder und sonstiges Drittmaterial unterliegen ebenfalls der genannten Creative Commons Lizenz, sofern sich aus der Abbildungslegende nichts anderes ergibt. Sofern das betreffende Material nicht unter der genannten Creative Commons Lizenz steht und die betreffende Handlung nicht nach gesetzlichen Vorschriften erlaubt ist, ist für die oben aufgeführten Weiterverwendungen des Materials die Einwilligung des jeweiligen Rechteinhabers einzuholen.

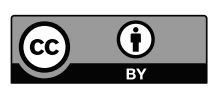

